\begin{tabular}{|l|l|l||}
\hline \multicolumn{2}{|c|}{ PublisherInfo } \\
\hline \hline PublisherName & $:$ & BioMed Central \\
\hline \hline PublisherLocation & $:$ & London \\
\hline \hline PublisherImprintName & $:$ & BioMed Central \\
\hline \hline
\end{tabular}

\title{
Suppression of ERK cascade by scaffold protein JSAP1
}

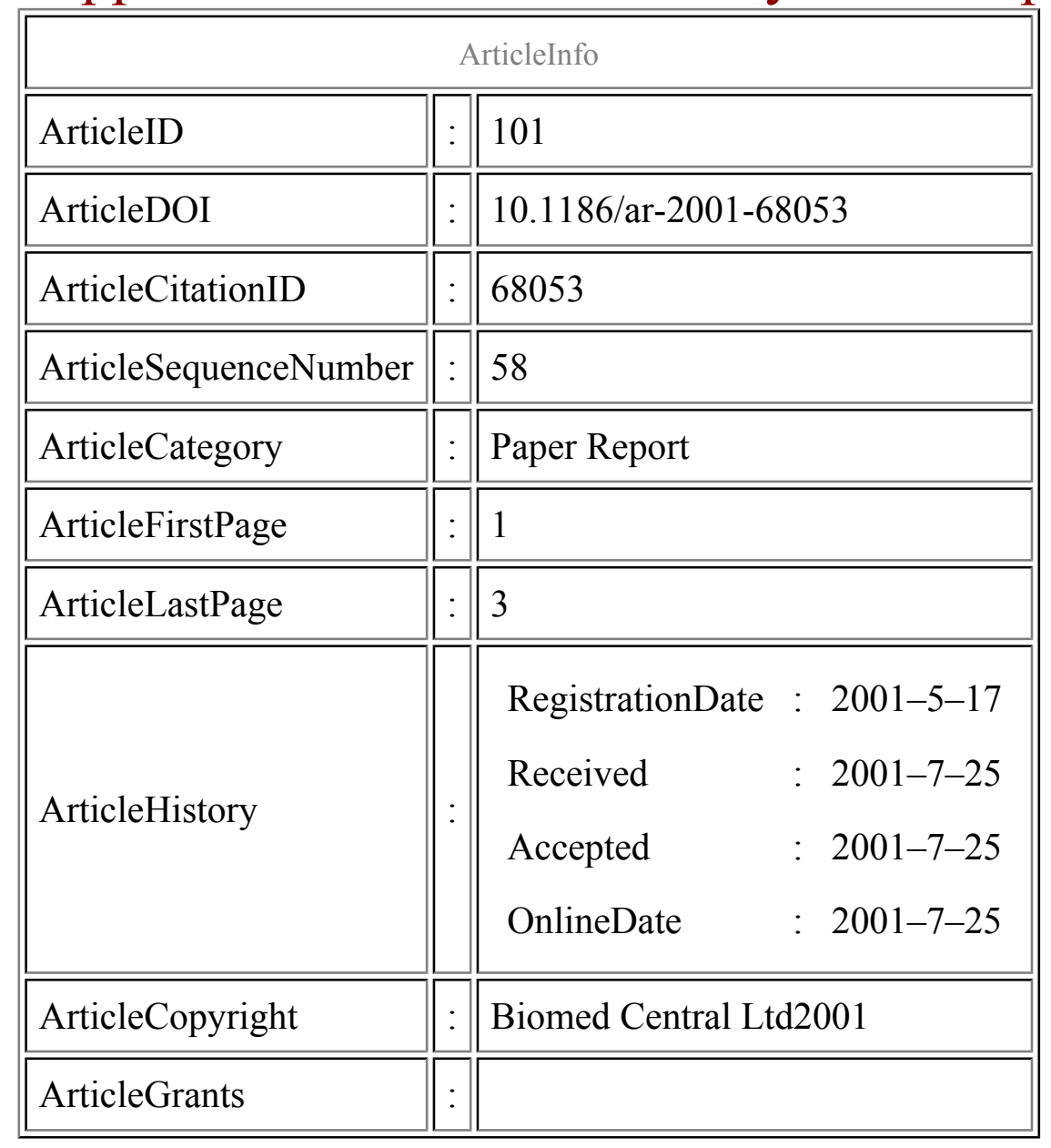




\begin{tabular}{|l|l|l|l|}
\hline ArticleContext & $:$ & 130753311 \\
\hline
\end{tabular}

Allan Sudlow, ${ }^{\text {Aff1 }}$

Aff1 The Kennedy Institute of Rheumatology, London, UK

Keywords

ERK, JSAP1, MAPK, scaffold protein, signal transduction

\section{Context}

The mitogen activated protein (MAP) kinase (MAPK) signalling pathway is an intracellular cascade, identified in organisms from yeast to mammals, consisting primarily of three components (MAPKKK $>$ MAPKK $>$ MAPK). In mammals, five such pathways have so far been identified, including the MEKK (MAP/extracellular signal related kinase [ERK] kinase kinase)-MKK4 (MAPK kinase)/7-cJun $\mathrm{NH}_{2}$ terminal kinase (JNK) cascade and Raf-MEK-ERK cascade. These cascades play key roles in many cellular processes, including responses to proinflammatory cytokines and environmental stresses. A family of scaffold proteins has recently emerged which appears to promote specific and efficient activation of these MAPK cascades. The scaffold protein JNK/stress-activated protein kinase-associated protein (JSAP)1 (JIP3) has previously been shown to enhance activation of the JNK pathway by binding its components. In the current study, JSAP1 is now also shown to actively suppress the Raf-MEK-ERK cascade.

\section{Significant findings}

JSAP1 prevents activation of ERK by phorbol ester (PMA), constitutively active Raf or Ras-activated Raf by interfering with the phosphorylation and activation of MEK1 by Raf. The carboxy-terminal kinase domain of Raf binds directly to the carboxyl terminus of JSAP1 and this is probably the mechanism of inhibition. JSAP1 may also bind MEK1 (indirectly) and thus may act like Raf kinase inhibitor protein, which dissociates a Raf-MEK1 complex and behaves as a competitive inhibitor of MEK phosphorylation.

\section{Comments}


In yeast, scaffold proteins have been shown to insulate against 'cross-talk' between different MAPK cascades to allow selective activation of shared kinases to elicit different responses. Uniquely, this paper demonstrates how a mammalian scaffold protein may suppress activation of one MAPK pathway (ERK) as well as, on past evidence, enhance activation via another MAPK pathway (JNK). However, this is not shown in tandem. Also, although JSAP1 binding to endogenous Raf is demonstrated, the majority of the experiments involve overexpression of transfected proteins which have, in the past, led to initial characterisation of other MAPK scaffold proteins (JSAP1) as pathway inhibitors.

\section{Methods}

Transient transfection, protein binding, immunoblotting, kinase assay, autoradiography

\section{Additional information}

\section{References}

1. Kuboky Y, Ito M, Takamatsu N, Yamamoto K, Shiba T, Yoshioka K: A scaffold protein in the c-Jun $\mathrm{NH}_{2}$-terminal kinase signalling pathways suppresses the extracellular signal-regulated kinase signalling pathways. J Biol Chem. 2000, 275: 39815-39818.

This PDF file was created after publication. 